Bull. Austral. Math. Soc.

VOL. $72(2005) \quad[381-384]$

\title{
A CHARACTERISTIC SUBGROUP AND KERNELS OF BRAUER CHARACTERS
}

\author{
I.M. ISAacs AND Gabriel Navarro
}

\begin{abstract}
If $G$ is finite group and $P$ is a Sylow $p$-subgroup of $G$, we prove that there is a unique largest normal subgroup $L$ of $G$ such that $L \cap P=L \cap \mathrm{N}_{G}(P)$. If $G$ is $p$-solvable, then $L$ is the intersection of the kernels of the irreducible Brauer characters of $G$ of degree not divisible by $p$.
\end{abstract}

\section{InTRODUCTION}

Our aim in this note is to prove the following two results.

Theorem A. Let $G$ be an arbitrary finite group and let $P$ be a Sylow $p$-subgroup of $G$ for some prime $p$. Then there exists a unique largest normal subgroup $L$ of $G$ such that

$$
L \cap P=L \cap \mathbf{N}_{G}(P)
$$

Note that the intersection property in Theorem $\mathrm{A}$ is equivalent to saying that $\mathrm{N}_{L}(P)$ is a $p$-group. Also, since this property is clearly independent of the choice of $P$ in $\operatorname{Syl}_{p}(G)$, it is clear that $L$ is characteristic in $G$. Our interest in this characteristic subgroup was motivated by the following.

THEOREM B. Suppose that $G$ is p-solvable and let $L$ be the largest normal subgroup of $G$ such that $L \cap P=L \cap \mathrm{N}_{G}(P)$, where $P \in \operatorname{Syl}_{p}(G)$. Then $L$ is the intersection of the kernels of the irreducible Brauer characters of $G$ with degree not divisible by $p$.

The assumption that $G$ is $p$-solvable in Theorem B is essential. Consider, for example, the simple group $G=M_{23}$ and take $p=2$. Then $G$ has a self-normalising Sylow 2-subgroup, and thus the characteristic subgroup $L$ of Theorem $A$ is the whole group $G$. But $G$ has an irreducible Brauer character of degree 11, and hence the conclusion of Theorem B fails in this case.

Received 8th June, 2005

The second author is partially supported by the Ministerio de Educación y Ciencia proyecto MTM200406067-C02-01.

Copyright Clearance Centre, Inc. Serial-fee code: 0004-9727/05 \$A2.00+0.00. 


\section{PROOFS}

Theorem $\mathrm{A}$ is an immediate consequence of, [4, Lemma 5.3], and so we take this opportunity to offer a new and simpler proof of a somewhat more general result. The original lemma is the case of the following where both $H$ and $K$ are normal in $G$.

Lemma 2.1. Let $G$ be a finite group and let $P \in \operatorname{Syl}_{p}(G)$, where $p$ is a prime. Let $H$ and $K$ be subgroups of $G$ such that $H K, H P$ and $K P$ are subgroups. Then

$$
\mathbf{N}_{H K}(P)=\mathbf{N}_{H}(P) \mathbf{N}_{K}(P) .
$$

Proof: We argue by induction on $|G: H||G: K|$. Note that $|H: P \cap H|=\mid H P$ : $P \mid$ is coprime to $p$, and so $P \cap H$ is a Sylow $p$-subgroup of $H$ and similarly, $P \cap K$ is a Sylow $p$-subgroup of $K$. It follows that

$$
|(P \cap H)(P \cap K)|=\frac{|P \cap H||P \cap K|}{|P \cap H \cap K|} \geqslant \frac{|H|_{p}|K|_{p}}{|H \cap K|_{p}}=|H K|_{p} \geqslant|P \cap H K|,
$$

and thus $(P \cap H)(P \cap K)=P \cap H K$.

Suppose first that $P$ is not contained in $H$. We can then apply the inductive hypothesis with $P H$ in place of $H$, and we deduce that

$$
\mathbf{N}_{P H K}(P)=\mathbf{N}_{P H}(P) \mathbf{N}_{K}(P) .
$$

By Dedekind's lemma, $\mathrm{N}_{P H}(P)=\mathrm{N}_{H}(P) P$, and thus

$$
\mathbf{N}_{P H K}(P)=\mathbf{N}_{H}(P) P \mathbf{N}_{K}(P) .
$$

Now let $g \in \mathrm{N}_{H K}(P)$. We can then write $g=x u y$, where $x \in \mathrm{N}_{H}(P), u \in P$ and $y \in \mathbf{N}_{K}(P)$. Since $g, x$ and $y$ are all in $H K$, we see that also $u \in H K$, and therefore $u \in P \cap H K$. By the first paragraph, we can write $u=r s$, where $r \in P \cap H$ and $s \in P \cap K$. Then

$$
g=(x r)(s y) \in \mathrm{N}_{H}(P) \mathbf{N}_{K}(P),
$$

and we are done in this case. Similarly the lemma is proved if $P$ is not contained in $K$.

We can now assume $P$ is contained in $H \cap K$, and we denote this intersection by $D$. Suppose that $g \in \mathbf{N}_{H K}(P)$ and write $g=h k^{-1}$, with $h \in H$ and $k \in K$. Since $P^{g}=P$, we have $P^{k}=P^{h}$ and this subgroup is contained in both $H$ and $K$. By Sylow's theorem in the group $D=H \cap K$, we have $P^{h}=P^{d}$ for some element $d \in D$, and thus $h d^{-1} \in \mathbf{N}_{H}(P)$. Also $P^{k}=P^{d}$, so $d k^{-1} \in \mathbf{N}_{K}(P)$. We see now that

$$
g=\left(h d^{-1}\right)\left(d k^{-1}\right) \in \mathbf{N}_{H}(P) \mathbf{N}_{K}(P),
$$

and the proof is complete.

Now we are ready to prove Theorem A. 
Proof of Theorem A: Let $P \in \operatorname{Syl}_{p}(G)$, and write $N=\mathbf{N}_{G}(P)$. Suppose that $H$ and $K$ are normal subgroups of $G$, each maximal with the property that its intersection with $N$ is equal to its intersection with $P$. We must show that $H=K$. By Lemma 2.1. we have

$$
N \cap H K=\mathbf{N}_{H K}(P)=\mathbf{N}_{H}(P) \mathbf{N}_{K}(P) .
$$

Then $N \cap H K$ is a product of two $p$-subgroups, and so it is a $p$-subgroup of $N$. Since $P$ is the unique Sylow $p$-subgroup of $N$, it follows that $N \cap H K=P \cap H K$. Now by the maximality of $H$ and $K$, we conclude that $H=H K=K$, and the proof is complete.

To prove Theorem B, we choose to work with the $p^{\prime}$-special characters of the $p$ solvable group $G$. (Their properties can be found in [1]. In particular, these members of $\operatorname{Irr}(G)$ form a set of lifts for the irreducible Brauer characters of $G$ having $p^{\prime}$-degree.)

THEOREM 2.2. Let $G$ be a p-solvable group and let $K$ be the intersection of the kernels of the $p^{\prime}$-special characters of $G$. Then $K$ is the largest normal subgroup of $G$ such that $K \cap P=K \cap \mathrm{N}_{G}(P)$, where $P \in \operatorname{Syl}_{p}(G)$.

Proof: Write $N=\mathrm{N}_{G}(P)$. First, we prove by induction on $|G|$ that $K \cap P$ $=K \cap N$. We may assume that $K>1$, and we choose a minimal normal subgroup $M$ of $G$ with $M \subseteq K$. Now, $K / M$ is the intersection of the kernels of the $p^{\prime}$-special characters of $G / M$ and $P M / M$ is a Sylow $p$-subgroup of $G / M$ with normaliser $N M / M$. By the inductive hypothesis, we deduce that

$$
(K / M) \cap(N M / M)=(K / M) \cap(P M / M),
$$

or equivalently, $K \cap N M=K \cap P M$. If $M$ is a $p$-group, then $P M=P$ and $N M=N$, and we are done in this case. We may therefore assume that $M$ is a $p^{\prime}$-group. Since $M \subseteq K$, Dedekind's lemma yields that

$$
(K \cap P) M=K \cap P M=K \cap N M=(K \cap N) M .
$$

and therefore, if we can show that $(K \cap P) \cap M=(K \cap N) \cap M$, it will follow that $|K \cap P|=|K \cap N|$, and thus $K \cap P=K \cap N$, as required. In particular, since $M \subseteq K$, it suffices to show that $N \cap M=1$. As $M$ is a normal $p^{\prime}$-subgroup of $G$, it follows that $N \cap M=\mathbf{C}_{N}(P)$, and if this is nontrivial, then by the Glauberman character correspondence, (see [3, Chapter 13]), there exists a nonprincipal $P$-invariant character $\theta \in \operatorname{Irr}(M)$. Then there exists a $p^{\prime}$-special character $\chi \in \operatorname{Irr}(G)$ lying over $\theta$ by $[1$, Corollary (4.8)]. However, $M \subseteq K \subseteq \operatorname{ker}(\chi)$ and this is a contradiction.

Finally, we need to show that if $K<L \triangleleft G$, then $L \cap P<L \cap N$, and for this purpose, we can assume that $L / K$ is a chief factor of $G$. Assuming that $L \cap N=L \cap P$, we work to derive a contradiction. Since $K<L$, there exists a $p^{\prime}$-special character $\chi \in \operatorname{Irr}(G)$ such that $L$ is not contained in $\operatorname{ker}(\chi)$. But $\chi$ has $p^{\prime}$-degree, and this implies that $\chi_{L}$ has a nonprincipal $P$-invariant irreducible constituent $\theta$, and $\theta$ is necessarily $p^{\prime}$-special 
since it lies under $\chi$. Also, $K \subseteq \operatorname{ker}(\theta)$, and thus $L / K$ cannot be a $p$-group because it has a nonprincipal $p^{\prime}$-special character. We deduce that $L / K$ is a $p^{\prime}$-group, and thus $L \cap N=L \cap P \subseteq K$ and we have $L \cap N K=(L \cap N) K=K$. Observe, however, that $N K / K$ is the full normaliser of $P K / K$ in $G / K$, and so it follows that $\mathrm{C}_{L / K}(P)$ is trivial. By the Glauberman correspondence, however, $\mathrm{C}_{L / K}(P)$ must be nontrivial since $L / K$ has a nonprincipal $P$-invariant irreducible character. This is a contradiction and the theorem is proved.

Finally, we complete the proof of Theorem B.

Proof of Theorem B: By [2, Lemma (5.4) and Corollary (10.3)], we know that restriction to $p$-regular elements defines a bijection from the set of $p^{\prime}$-special characters of $G$ onto the irreducible Brauer characters of $G$ having $p^{\prime}$-degree. It follows that the intersection $K$ of the kernels of all $p^{\prime}$ - special characters of $G$ is contained in the intersection $L$ of the kernels of all irreducible Brauer characters having $p^{\prime}$-degree. By Theorem 2.2, therefore, it suffices to show that $L=K$.

Every $p$-regular element of $L$ must lie in $K$, and thus $L / K$ is a $p$-group. By Theorem 2.2 , we know that $K \cap N=K \cap P$, where $P \in \operatorname{Syl}_{p}(G)$ and $N=\mathrm{N}_{G}(P)$. As $N \cap K$ is a $p$-group and $L / K$ is a $p$-group, it is easy to see that $N \cap L$ is also a $p$-group, and thus $N \cap L=P \cap L$. By the maximality of $K$ in Theorem 2.2, we conclude that $L=K$, as desired.

\section{REFERENCES}

[1] D. Gajendragadkar, 'A characteristic class of characters of finite $\pi$-separable groups', $J$. Algebra 59 (1979), 237-259.

[2] I.M. Isaacs, 'Characters of $\pi$-separable groups', J. Algebra 86 (1984), 98-112.

[3] I.M. Isaacs, Character theory of finite groups (Dover Publication, New York, 1994).

[4] G. Navarro, 'A new character correspondence in groups of odd order', J. Algebra 268 (2003), 8-21.

Department of Mathematics

University of Wisconsin

Madison, WI 53706

United States of America

e-mail: isaacs@math.wisc.edu
Facultat de Matemàtiques

Universitat de València

Burjassot, València 46100

Spain

e-mail: gabriel@uv.es 